\title{
Improve the material absorption of light and enhance the laser tube bending process utilizing laser softening heat treatment
}

\begin{abstract}
Laser forming is a flexible control process that has a wide spectrum of applications; particularly, laser tube bending. It offers the perfect solution for many industrial fields, such as aerospace, engines, heat exchangers, and air conditioners. A high power pulsed Nd-YAG laser with a maximum average power of $300 \mathrm{~W}$ emitting at $1064 \mathrm{~nm}$ and fiber-coupled is used to irradiate stainless steel 304 (SS304) tubes of $12.7 \mathrm{~mm}$ diameter, $0.6 \mathrm{~mm}$ thickness and $70 \mathrm{~mm}$ length. Moreover, a motorized rotation stage with a computer controller is employed to hold and rotate the tube. In this paper, an experimental investigation is carried out to improve the laser tube bending process by enhancing the absorption coefficient of the material and the mechanical formability using laser softening heat treatment. The material surface is coated with an oxidization layer; hence, the material absorption of laser light is increased and the temperature rapidly rises. The processing speed is enhanced and the output bending angle is increased to $1.9^{\circ}$ with an increment of $70 \%$ after the laser softening heat treatment.
\end{abstract}

Keyword: Laser forming; Laser tube bending; Laser softening heat treatment; Material absorptionl. 